\title{
HISTOMORPHOMETRIC ASSESSMENT OF AUTOGENOUS STEM CELL THERAPY IN HORIZONTAL ALVEOLAR RIDGE AUGMENTATION (EXPERIMENTAL STUDY)
}

\author{
Gillan I. El-Kimary ${ }^{1}{ }_{M S c}$, Ahmed M. Hommos ${ }^{2} P h D$, Mohamed I. Sayed ${ }^{3} P h D$, \\ Gehan S. Kotry ${ }^{2} P h D$, Dina A. Nagui ${ }^{4} P h D$.
}

\begin{abstract}
INTRODUCTION: Augmentation of deficient alveolar ridge is a challenging task in periodontal regenerative therapy. The gold standard therapeutic modality for managing these defects is autogenous bone grafting. But, this approach was hampered by increased patient morbidity. Thus, recent advances in molecular and cellular biology have shed light on the "bioengineering" technology which seeks to introduce the incorporation of stem cells in periodontal regeneration.

OBJECTIVE: The aim of this experimental study was to evaluate, histomorphometrically, the efficacy of guided bone regeneration (GBR) technique using autogenous bone marrow-derived mesenchymal stem cells (BMSCs) loaded on $\beta$-tricalcium phosphate ( $\beta$-TCP) alloplast as a scaffold, in managing horizontal alveolar ridge defects.

MATERIALS AND METHODS: Sixteen critical-sized horizontal ridge defects were created in four healthy mongrel dogs after bilateral extraction of the mandibular third and fourth premolars. In a split mouth design; the experimental group (right side): comprised eight ridge defects managed using GBR approach (autogenous BMSCs $+\beta$-TCP + collagen membrane). The control group (left side): comprised eight ridge defects were left empty to heal spontaneously. Dogs were sacrificed at 1 and 2 months intervals for histomorphometric assessment using Image J software to measure osteoblastic count (OC) from the obtained histological sections.

RESULTS: The experimental group showed a statistically significant increase in OC at both follow up intervals of the study.

CONCLUSIONS: GBR technique using autogenous BMSCs/ $\beta$-TCP composites was effective in managing horizontal ridge defects. Osteogenic properties and bone regenerative capacity of alloplasts can be greatly enhanced using this technique in periodontal regenerative surgeries.

KEY WORDS: Alveolar ridge defects; Autogenous stem cells; Guided bone regeneration; Osteoblastic count

RUNNING TITLE: Autogenous stem cell therapy in horizontal ridge augmentation.
\end{abstract}

1 Assistant lecturer, Department of Oral Medicine, Periodontology, Oral Diagnosis and Radiology, Faculty of Dentistry, Alexandria University

2 Professor, Department of Oral Medicine, Periodontology, Oral Diagnosis and Radiology, Faculty of Dentistry, Alexandria University

3 Professor, Department of Clinical Pathology, Faculty of Medicine, Alexandria University

4 Assistant Professor, Department of Oral Biology, Faculty of Dentistry, Alexandria University

* Corresponding Author:

Email: gillan.elkimary@gmail.com

\section{INTRODUCTION}

Implant-supported prostheses for functional and esthetic rehabilitation of tooth loss have become a widely used established treatment modality in modern dentistry (1). However, placement of dental implants and esthetic outcomes are sometimes compromised by the presence of large alveolar ridge defects. Consequently, bone volume at the future implant site is considered to be one of the pivotal factors in overall treatment planning $(1,2)$.

The quantity and quality of the existing alveolar bone available in all spatial dimensions affect the success of implant placement by influencing three important aspects; implant osseointegration, the outline of the soft tissue and prosthetic parameters such as the axis of implant and loaded restoration $(1,2)$.

The remodeling process following tooth extraction results in significant resorptive changes particularly on the buccal aspect. These changes include both soft tissue collapse and volumetric alveolar ridge loss in both apico-coronal and bucco-lingual dimensions resulting in a ridge morphology located more palatal or lingual in comparison to the preextraction tooth position (3-5).

A multitude of surgical techniques have been proposed for bone augmentation of small/medium horizontal alveolar ridge defects $(1,6)$. These techniques include: GBR with or without tenting approach, ridge expansion procedures with 
osteotomes or osseodensification techniques, ridge splitting piezoelectric surgical approach, and distraction osteogenesis (7-9). Mesenchymal stem cells (MSCs) belong to adult stem cells that are present in several mesenchymal tissues. These cells possess two main features; firstly, self-renewal by dividing and secondly, multi-lineage differentiation capacity into multiple tissues such as bone, cartilage, muscle or fat tissue (10-13). The osteogenic potential of MSCs to differentiate into osteoblasts provides the basic characteristic aspects of osteoinduction and osteoconduction of autologous bone grafting, thereby obviating the associated harvesting complications $(2,14)$.

Bone marrow-derived MSCs (BMSCs) are a subpopulation of multipotent progenitor non-haematopoietic stromal cells residing in bone marrow. This group of stem cells can be expanded easily and differentiate into cells with an osteogenic phenotype (15). In addition to the iliac crest which is considered the main and most frequently used harvesting site, BMSCs can be isolated from other sources such as orofacial bones, including maxilla and mandible $(16,17)$.

Alloplasts are osteoconductive bone replacement grafts without an intrinsic potential for osteogenesis or osteoinduction. They serve as scaffolds in regenerative procedures and have no risk of disease transmission and no need of second surgical sites (18). $\beta$-tricalcium phosphate ( $\beta$ TCP) is a porous form of calcium phosphate that is widely used as a bone grafting material.

It has been shown that the rate of resorption of $\beta$-TCP is quite similar to the rate of apposition of new bone. Consequently, this graft material has the ability to act as a scaffold to induce osteoconduction of bone into the defect site, then it is totally resorbed. Thus, no biomaterial is permanently left within the reconstruction site $(18,19)$.

Since, enhancing the osteoinductive properties of bone substitutes is a necessity in managing periodontal defects, the present study attempts to test the effectiveness of the adjunctive use of autogenous BMSCs loaded on $\beta$-tricalcium phosphate $(\beta$-TCP) alloplast as a scaffold, in managing horizontal alveolar ridge defects in dogs.

The null hypothesis of the present experimental study presumes no significant difference in bone regeneration will be obtained using GBR approach (BMSCs/ $\beta$-TCP scaffold and collagen membrane) compared to the spontaneous healing of critical-sized horizontal ridge defects.

\section{MATERIALS AND METHODS}

Experimental study

The research protocol was approved by the Ethical Committee of the Alexandria University following the guidelines of the Institutional Review Boards of the Faculty of Dentistry, Alexandria University.

A total of four male mongrel dogs (Canis familaris), about 18-24 months old, weighting approximately $18-24 \mathrm{~kg}$, were included in this study. All dogs were systemically healthy with intact maxillary and mandibular dentitions. Animals were housed under the same conditions at the experimental animal house, Medical Research Institute, Alexandria University.

Study design and grouping
This study was conducted as a split mouth design. It included two groups:

1) Experimental group (right side): included eight criticalsized horizontal alveolar ridge defects that were managed with GBR using autogenous BMSCs, osteoconductive bone grafting material and collagen membrane (BMSCs/ $\beta$-TCP).

2) Control group (left side): included eight critical-sized horizontal alveolar ridge defects that were left empty to heal spontaneously.

\section{Surgical Procedure}

All surgical procedures were accomplished while animals were under general anesthesia using intravenous sodium thiopental (13mg/kg (Sandoz GmbH, Kundl, Austria)). The slow intravenous solution was administered through the cephalic vein with a $25 \times 8-\mathrm{cm}$ needle.

A- Sampling and BMSCs isolation (20)

One day before surgery, heparinized bone marrow aspirates of $10 \mathrm{ml}$ were aspirated from the iliac crest of each animal using sterile wide bone marrow aspiration needles. In a laminar air flow hood, the samples were drawn in falcon tubes with an equal volume of DMEM low glucose solution (DMEM Low Glucose, Biowest ${ }^{\circledR S A S}$, Nuaillé, France). The falcon tubes were then centrifuged at $1200 \mathrm{rpm}$ for 10 minutes and the middle buffy coat layer (contains mostly granulocytes and mononuclear cells which were needed for implantation with the $\beta$-TCP scaffold) was carefully aspirated and collected in another falcon tube.

The collected mononuclear cell fraction was washed again by DMEM low glucose solution and centrifuged at $2000 \mathrm{rpm}$ for 10 minutes, 3 successive times. The resultant mononuclear cell pellets were resuspended in the DMEM low glucose cell culture medium to be introduced into the scaffold ( $\beta$-TCP (Adbone ${ }^{\circledR}$ TCP, Medbone ${ }^{\circledR}$ Sintra, Portugal)) in a sterile container. In CO2 incubator (Air-Jacketed, NU-5100E/G DH automatic $\mathrm{CO} 2$ incubator, MA, USA), the grafting materials were incubated for 24 hours before implantation to allow for cell attachment.

B- Creation of ridge defects

Sulcular incisions were taken and mucoperiosteal flaps were reflected buccally at the mandibular third and fourth premolar region, bilaterally. A periosteal incision was done at the base of each flap to aid in coverage and tension free closure. Mandibular third and fourth premolar teeth (P3 and P4) were extracted bilatellary, either as one unit or sectioned into two halves when needed. In each dog, four critical-sized horizontal alveolar ridge defects were created preserving the lingual walls. The dimensions of all defects were standardized to be about $7 \mathrm{~mm}$ mesio-distally, $8 \mathrm{~mm}$ apicocoronally and $5 \mathrm{~mm}$ bucco-lingually $(21,22)$. With the aid of rotary burs, bone was removed with thourough irrigation using sterile saline to avoid overheating. The right side experimental defects were managed with GBR technique using autogenous BMSCs cells, alloplast. (BMSCs/ $\beta$-TCP scaffold). The left control side defects were left empty to heal spontaneously. Collagen membranes were trimmed with the same configuration and adapted to cover the experimental defect areas extending to the surrounding bone by $2-3 \mathrm{~mm}$. Flaps were coronally positioned and closure of the wound 
area was performed with interrupted suturing, using 3-0 silk suture (Ethicon silk suture, Johnson \& Johnson, Somerville, New Jersy, USA).

\section{Postoperative care}

All animals received: Antibiotic (Ampicillin 1gm, Eipico, Egypt), 1g. It was administered via intramuscular route in the first day. Afterwards, it was added to dogs food for seven days. Non-steroidal anti- inflammatory (Brufen $600 \mathrm{mg}$, Abbot GmbH, Germany) was also given intravenous the first day postoperative. The dogs were only served with soft diet during the healing period to reduce occlusal forces and trauma to the surgical site. The sutures were removed 10 days, post-operatively.

Animals sacrifice

The dogs were sacrificed according to the following order: 2 dogs, 1 month after surgery. The remaining 2 dogs were sacrificed 2 months after surgery. This was accomplished with an intravenous overdose injection of anesthesia (concentrated sodium thiopental).

Preparation of histologic sections

The mandibles were dissected and sectioned into two halves, then placed in a fixative of $10 \%$ buffered neutral formalin for two days, decalcified in multiple baths of $8 \%$ trichloroacetic acid. After decalcification, the blocks were immersed in paraffin, and semiserial $(4-5 \mu \mathrm{m})$ wide histologic sections in mesio-distal direction using a rotatory microtome were taken. Histologic sections were then stained with Hematoxylin and Eosin (H\&E) stain for general examination of the operating site tissues (23).

Histomorphometric quantitative analysis

Histologic photomicrographs of the H\&E stained sections (all obtained at x100 original magnification) were quantitatively analyzed of the regenerative process outcome using image analysis software (image J 1.46r software (24)). The osteoblastic count (parameter of interest) was measured for experimental and control groups at the two study observation periods after four and eight weeks of sacrifice. Measurements were finally averaged and standard deviation (SD) was calculated.

Osteoblastic count (OC) measurement using image J program The number of osteoblasts in each photomicrograph was calculated by free hand selection using the "multi-point" button. OC was repeated three times for each specimen and the average value was used to represent the final value for this specimen used in statistical analysis. (Figure $1 \mathrm{~A} \& \mathrm{~B}$ )

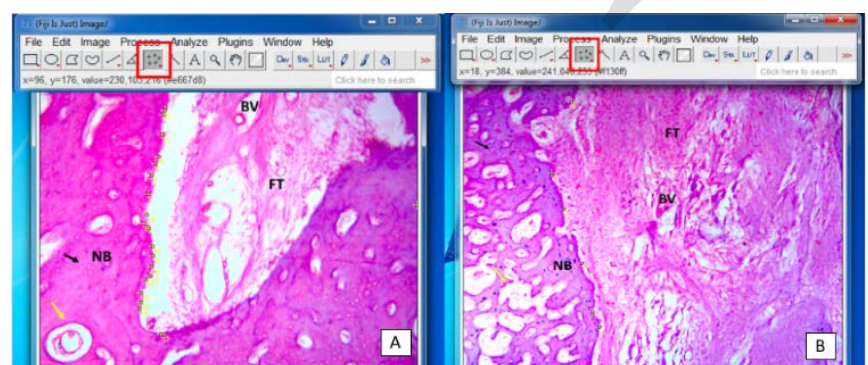

Figure 1: Osteoblastic cell count using image J program in the study groups. (A) Experimental group, and (B) Control group. (NB: new bone, FT: fibrous tissue, BV: blood vessel, black arrow: osteocytes, yellow arrow: bone marrow spaces, black empty circles: lining osteoblast cells, and red square: the "multi-point" button in the image J software).

\section{Statistical analysis}

Data was presented using mean and SD. Paired t-test was applied to compare between the experimental and control groups regarding the OC and for tracing changes across time. Significance level was set at $\mathrm{p}$ value $\leq 0.05$.

\section{RESULTS}

Histomorphometric analysis results (Osteoblastic Count) Quantitative assessment of the OC obtained from the histologic light micrographs of both study groups using image J software revealed the following:

The experimental group showed a statistically significant increase in OC compared to control group at both follow up time intervals of the study. The mean OC after one month in the experimental and control groups were 39.75 \pm 3.86 and $13.75 \pm 2.75$, respectively $(\mathrm{P}=0.001)$. After two months it was $17.75 \pm 4.11$ and $7.5 \pm 1.29$ for the same groups, respectively $(\mathrm{P}=0.014)$. (Table 1) (Figure 2)

Furthermore, there was a statistically significant decrease in osteoblastic count related to experimental group between both follow up intervals of the study. (Mean value was 39.75 \pm 3.86 after one month and $17.75 \pm 4.11$ after two months) $(\mathrm{P}=0.0003)$. Similarly, there was a statistically significant decrease in osteoblastic count related to control group between both follow up intervals of the study. (Mean value was $13.75 \pm 2.75$ after one month and $7.5 \pm 1.29$ after two months) $(\mathrm{P}=0.02)$

Moreover, when comparing the percent change in osteoblastic count across the study period from first to second follow up intervals between both experimental and control groups, there was a non-statistically significant reduction in experimental group when compared to the control group. (Mean value was $55.55 \pm 7.58$ and $43.18 \pm 16.86$ respectively) $(\mathrm{P}=0.174)$. (Table 2) (Figure 3)

Table 1: Comparison of osteoblast count between the experimental and control groups at different time points

\begin{tabular}{|c|c|c|c|}
\hline \multirow{2}{*}{} & $\begin{array}{c}\text { Experimental } \\
\text { group } \\
(\mathrm{n}=4)\end{array}$ & $\begin{array}{c}\text { Control } \\
\text { group } \\
(\mathrm{n}=4)\end{array}$ & \multirow{2}{*}{ P value } \\
\cline { 2 - 3 } & \multicolumn{2}{|c|}{ Mean (SD) } & \\
\hline 1 month & $39.75(3.86)$ & $13.75(2.75)$ & $0.001^{*}$ \\
\hline 2 months & $17.75(4.11)$ & $7.5(1.29)$ & $0.014^{*}$ \\
\hline P value & $0.0003^{*}$ & $0.02^{*}$ & \\
\hline
\end{tabular}

*Statistically significant difference at $p$ value $e \leq 0.05$

Table 2: Comparison of percent change in osteoblast count between the experimental and control groups after 2 months

\begin{tabular}{|l|c|c|c|}
\hline & $\begin{array}{c}\text { Experimenta } \\
\text { l group } \\
(\mathrm{n}=4)\end{array}$ & $\begin{array}{c}\text { Control group } \\
(\mathrm{n}=4)\end{array}$ & \multirow{2}{*}{ P value } \\
\cline { 2 - 3 } & \multicolumn{2}{|c|}{ Mean (SD) } & \\
\hline \% Reduction & $55.55(7.58)$ & $43.18(16.86)$ & 0.174 \\
\hline
\end{tabular}

*Statistically significant difference at $p$ value $e \leq 0.05$ 


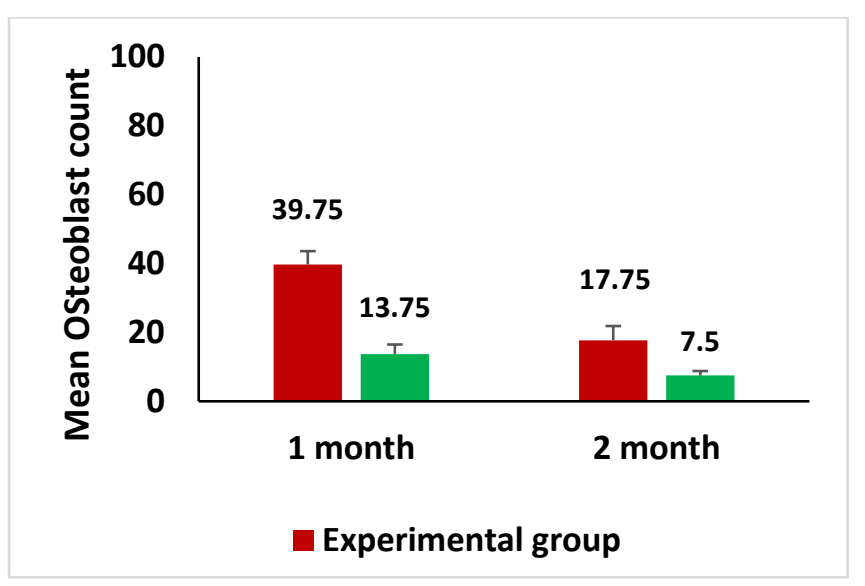

Figure 2: Mean osteoblast count in the experimental and control groups at different time points

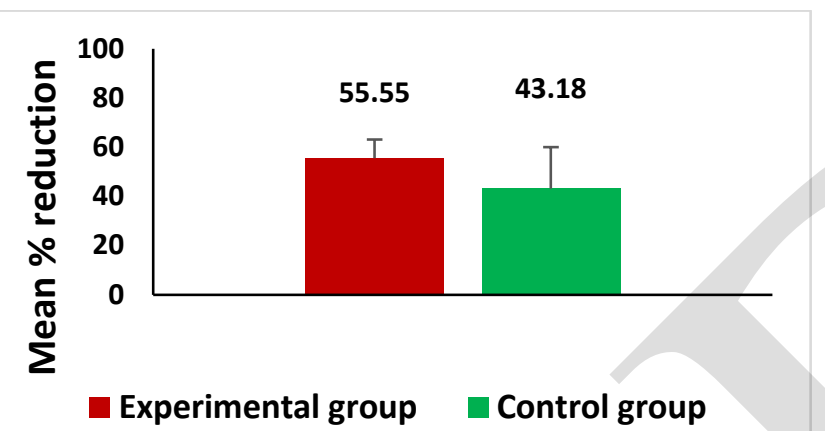

Figure 3: Mean percent reduction in osteoblast count between experimental and control groups after 2 months

\section{DISCUSION}

Management of alveolar ridge defects is considered a challenging surgical procedures in regenerative periodontal therapy. The defects formed after tooth extraction result in limitation of the ideal three dimensional placement of dental implants (25). Many surgical interventions have been described to augment atrophic ridges at future implant sites, overcoming the main problem of lack in adequate bone volume essential for implant stability (26).

Periodontal regeneration is the ultimate target of periodontal therapy. Bone regeneration can be improved by incorporating the concept of tissue engineering to enhance the quantity and quality of the newly formed bone. Because of the limitations of some current osteoconductive bone grafting materials, alternative methods for enhancing osteogenic properties of these materials in repairing bone defects were deemed necessary (27-29).

Mesenchymal stem cells (MSCs) play a pivotal role in many biological processes including wound healing, growth, replacement and repair of daily aged and exfoliated cells (30). BMSCs can be expanded easily in culture and differentiate into cells with an osteogenic phenotype (15). This was confirmed when these cells were implanted ectopically on an appropriate scaffold and bone formation was observed, making them an appropriate possible stem cell source for bone regeneration therapy $(12,31)$.
In addition to iliac crest, which is considered the main and most frequently used site in BMSCs harvesting for bone regeneration over the years (16), BMSCs can be isolated from many dental and non-dental sources such as orofacial bones, including maxilla and mandible. The choice of iliac crest to be the harvesting site in this study can be explained mainly by the abundance and large sample volume that could be aspirated. Furthermore, superior proliferation and osteogenic differentiation ability and lower adipogenic potential are other factors that were considered (32-35).

Despite the fact that BMSCs contain many osteoinductive growth factors and possess high osteogenic properties, they lack the osteoconductive scaffold features $(20,36)$. The choice of $\beta$-TCP as a scaffold grafting material in this study can be explained by many aspects. Firstly, when compared to hydroxyapatite, $\beta$-TCP has good resorbability in animal and human subjects. Secondly, high biocompatibility and possible promotive effects of $\beta$-TCP in the differentiation of BMSCs to osteoblasts. Moreover, the porous nature (up to $70 \%$ porosity) and high connectivity could play an important role in better inter-cellular communication (BMSCs and osteocytes) (20,37).

In the present study, sixteen critical-sized horizontal alveolar ridge defects have been surgically created in four healthy male mongrel dogs. In a split mouth design, the study included two groups as follows: the experimental group (right side): comprised eight ridge defects that were managed using GBR approach (autogenous BMSCs isolated from bone marrow aspirates one day before the surgery $+\beta$-TCP + collagen membrane). The control group (left side): comprised eight ridge defects the were left empty to heal spontaneously. The aim of this study was to assess histomorphometrically, the efficacy of GBR technique in managing alveolar ridge defects using autogenous BMSCs loaded on $\beta$-TCP alloplast as a scaffold grafting material.

Since most of the previous studies evaluated alveolar bone regeneration through clinical or radiographic assessments, we chose to conduct a histomorphometric analysis (in terms of osteoblastic cell counts). This provided us with a definitive insight on the level of osteogenesis process and bone formation in the created ridge defects.

The current study results demonstrated that critical-sized horizontal ridge defects can be repaired successfully with the (BMSCs/ $\beta$-TCP) construct. Bone formation was dramatically enhanced and almost complete bone fill was noticed in the experimental group two months postoperatively. Meanwhile, defects that were left empty in the control group have not been repaired spontaneously. These observations were directly correlated with the count of osteoblasts in histological specimens that truly reflect the activity of osteogenesis.

These findings are in agreement with several investigators. Yuan et al. (38) evaluated the management of segmental mandibular defects by bone graft construct (BMSCs seeded on porous $\beta$-TCP). Histologic and radiographic examinations showed that marked new bone formation and successful repair of such defects with complete union of bone was accomplished after 32 weeks in the experimental animals. The authors claimed that biodegradable scaffolds were 
essential in regeneration of new bone, since they provide the skeleton that creates spaces supporting the ingrowth of osteogenic cells and newly formed bone (38).

Our results are also in line with another experimental study conducted by Neamat et al. (39) They assessed the effect of pure phase $\beta$-TCP alloplast on osteogenesis and proliferation markers when incubated with BMSCs in intrabony osseous defects in a canine model. They concluded that overall new bone regeneration and osteogenesis were notably induced in the defects. They explained the obtained results by the possible proliferative effect of $\beta$-TCP on BMSCs by induction of the proliferating cell nuclear antigen (PCNA) and increased production of alkaline phosphatase, that dramatically increased the rate of differentiation of BMSCs into the osteogenic cell line.

Another explanation that could support the fact behind the differentiation of BMSCs into particular cells is the surrounding environment $(37,40)$. When BMSCs are transplanted adjacent to existing alveolar bone, definitive signals that could promote the differentiation of BMSCs into osteoblasts are delivered through the native bone matrix proteins and growth factors. Consequently, lower level of such signals is predicted to be received in areas far from the native alveolar bone (37). This speculation may not only explain our results in study group, but also justifies the presence of limited areas on new bone in the control group next to the native surrounding bone in the surgically created defects.

An additional finding in the current histomorphometric analysis was a reduction in osteoblastic count in both experimental and control groups across the study period from first to second follow up intervals. It is quite clear that there is an indirect relationship between osteoblastic count and the degree of bone maturation over time (29). This finding is in accordance with another immunohistopathologic study conducted by Afifi et al. (29) in a canine model with different periodontal defects. The authors evaluated the efficacy of guided tissue regeneration approach in managing furcation involvement defects. In line with the current study findings, their results depicted a decrease in osteoblastic count over time (one and two months intervals) with increasing bone maturity.

Based on the obtained results from the current work, BMSCs/ $\beta$-TCP composites increased osteoblastic activity in terms of proliferation (increased osteoblastic cell count) and subsequent new bone formation in critical-sized horizontal ridge defects. Further immunohistopathologic studies investigating the possible role of specific bone proteins over longer periods of time are highly recommended.

\section{CONCLUSIONS}

Within the limitations of the present work; it could be concluded that engineered bone (BMSCs loaded on $\beta$-TCP scaffold) was effective in horizontal alveolar ridge augmentation. Such approach is predictable in both, restoring and preserving ridge defects. Incorporating autogenous stem cells is also expected to improve the clinical outcomes in challenging large osseous defects and improve the osteoinductivity of osteoconductive bone substitutes.

\section{CONFLICT OF INTERESTS}

The authors declare that they have no conflict of interest. FUNDING

This research received no specific funding.

\section{REFERENCES}

1. Arx TV, Cochran DL, Hermann JS, Schenk R, Higginbottom FL, Buser D. Lateral ridge augmentation and implant placement: An experimental study evaluating implant osseointegration in different augmentation materials in the canine mandible. Int J Oral Maxillofac Implants. 2001;16:343-54.

2. Alvira-González J, Reina $M$, Sanche-Garces $M$. Assessment of bone regeneration using adipose-derived stem cells in critical size alveolar ridge defects: an experimental study in a dog model. Int J Oral Maxillofac Implants. 2016;31:1-9.

3. Schnutenhaus S, Götz W, Dreyhaupt J. Alveolar ridge preservation: A histomorphometric analysis. Open Dent J. 2018;12:916-28.

4. Moreno AR, Magdaleno MO, Islas MM, Mercado JA, Cruz ER. Postextraction alveolar preservation and use of the crown of the extracted tooth as a temporary restoration. Case Rep Dent. 2019;3:4262067.

5. Pagni G, Pellegrini G, Giannobile WV, Rasperini G. Postextraction alveolar ridge preservation: biological basis and treatments. Int J Dent. 2012;2012:151030.

6. Wang S, Zhang Z, Zhao J, Zhang X, Sun X. Vertical alveolar ridge augmentation with $\beta$-tricalcium phosphate and autologous osteoblasts in canine mandible. Biomaterials. 2009;30:2489-98.

7. Tian JH, Neiva R, Coelho PG, Witek L, Tovar NM. Alveolar ridge expansion: comparison of osseodensification and conventional osteotome techniques. J Craniofac Surg. 2019;30:607-10.

8. Moro A, Gasparini G, Foresta E, Saponaro G, Falchi M. Alveolar ridge split technique using piezosurgery with specially designed tips. Biomed Res Int. 2017;2017:4530378.

9. Shukla A, Singh SV, Kumar S, Mehrotra D, Mohammad S. Alveolar ridge augmentation using distraction osteogenesis: a clinical trial. J Oral Biol Craniofac Res. 2012;2:25-9.

10. Mahla R. Stem cells application in regenerative medicine and disease threpeutics. Int J Cell Biol. 2016;7:1-19.

11. Porcellini A. Regenerative medicine: A review. Rev Bras Hematol Hemoter. 2009;31:63-6.

12. Kanwal A, D’souza J, Muthiah L, Sirividya S. The current status of stem cell regeneration in intra oral applicationsa systematic review. OJST. 2017;7:197-224.

13. Ahn H, Kim K, Lee J. In vivo osteogenic differentiation of human adipose-derived stem cells in an injectable in situ-forming gel scaffold. Tissue Eng Part A. 2009;15:1821-32.

14. Ito K, Yamada Y, Nakamura S, Ueda M. Osteogenic potential of effective bone engineering using dental pulp stem cells, bone marrow stem cells, and periosteal cells for osseointegration of dental implants. Int $\mathrm{J}$ Oral Maxillofac Implants. 2011;26:947-54. 
15. Kim SH, Kim KH, Seo BM, Koo KT. Alveolar bone regeneration by transplantation of periodontal ligament stem cells and bone marrow stem cells in a canine periimplant defect model: a pilot study. J Periodontol. 2009;80:1815-23.

16. Paz A, Maghaireh H, Mangano F. Stem cells in dentistry: Types of intra- and extraoral tissue-derived stem cells and clinical applications. Stem Cells Int. 2018;2018:4313610.

17. Mueller S, Glowacki J. Age-related decline in the osteogenic potential of human bone marrow cells cultured in three-dimensional collagen sponges. J Cell. 2001;82:583-90.

18. Sheikh Z, Sima C, Glogauer M. Bone replacement materials and techniques used for achieving vertical alveolar bone augmentation. Materials. 2015;8:2953-93.

19. AlGhamdi AS, Shibly O, Ciancio SG. Osseous grafting part II: xenografts and alloplasts for periodontal regeneration-a literature review. J Int Acad Periodontol. 2010;12:39-44.

20. Eissa A, Karam S, Omar S, Sayed M. Effect of autogenous bone marrow mesenchymal stem cells in healing of mandibular osseous defects grafted with beta tricalcium phosphate in dogs. Alex Dent J. 2018;43:48-52.

21. Anwar S, Lotfy M, Kotry GS, Karam S. Efficacy of using platelet concentrates in the management of horizontal ridge defects (experimental study). Alex Dent J. 2018;43:99-104.

22. Sato I, Akizuki T, Oda S, Tsuchioka H, Hayashi C, Takasaki A. Histological evaluation of alveolar ridge augmentation using injectable calcium phosphate bone cement in dogs. J Oral Rehabil. 2009;36:762-9.

23. Drury RAB, Wallington EA, Carlton's histological techniques. 5th ed. Oxford: Oxford University Press; 1980.

24. Abramoff MD, Magalhaes PJ, Ram SJ. Image processing with Image J. Biophotonics International. 2004;11:36-42.

25. Amr A, Abdel Ghaffar K, Abuel-Ela H, Abd Elhamid E. Xenogenic flexible bone lamina graft: a successful alternative to the autogenous onlay bone block graft in alveolar ridge augmentation: A clinical, radiographic and histological evaluation. J Dent Treat Oral Care. 2017;1:104-17.

26. Woo R, Kim H, Kim G, Park W, Sohn D. Simplified 3Dimensional ridge augmentation using a tenting abutment. Adv Dent Oral Health. 2020;12:185-205.

27. Barradas A, Yuan H, Blitterswijk C, Habibovic P. Osteoinductive biomaterials: current knowledge of properties, experimental models and biological mechanisms. Eur Cell Mater. 2011;21:407-29.

28. Pradeep AR, Priyanka N, Kalra N, Savitha B. Clinical efficacy of subgingivally delivered 1.2-mg Simvastatin in the treatment of individuals with grade II furaction defects a randomized controlled clinical trial. J Periodontol. 2012;83:1472-9.
29. Afifi MM, Kotry GS, El-Kimary GI, Youssef HA. Immunohistopathologic evaluation of Drynaria fortunei rhizome extract in the management of Class II furcation defects in a canine model. J Periodontol. 2018;89:1362-71.

30. Hernández-Monjaraz B, Santiago-Osorio E, MonroyGarcía A, Ledesma-Martínez E, Mendoza-Núñez V. Mesenchymal stem cells of dental origin for inducing tissue regeneration in periodontitis: A mini-review. Int J Mol Sci. 2018;19:944-57.

31. Boo JS, Yamada Y, Okazaki Y, Hibino Y, Okada K. Tissue-engineered bone using mesenchymal stem cells and a biodegradable scaffold. J Craniofac Surg. 2002;13:231-9.

32. Matsubara T, Suardita K, Ishii M. Alveolar bone marrow as a cell source for regenerative medicine: differ- ences between alveolar and iliac bone marrow stromal cells. J Bone Miner Res. 2005;20:399-409.

33. Crespi R, Vinci R, Cappare P, Gherlone E, Romanos G. Calvarial versus iliac crest for autologous bone graft material for a sinus lift procedure: a histomorphometric study. Int J Oral Maxillofac Implants. 2007;22:527-32.

34. Borstlap W, Heidbuchel K, Freihofer H, KuijpersJagtman A. Early secondary bone grafting of alveolar cleft defects. A comparison between chin and rib grafts. J Craniomaxillofac Surg. 1990;18:201-5.

35. Akintoye S, Lam T, Shi S, Brahim J, Collins M, Robey P. Skeletal site-specific characterization of orofacial and iliac crest human bone marrow stromal cells in same individuals. Bone. 2006;38:758-68.

36. Russmueller G, Moser D, Spassova E, Plasenzotti R, Poeschl P, Seemann R. Tricalcium phosphate-based biocomposites for mandibular bone regeneration-A histological study in sheep. J Craniomaxillofac Surg. 2015;5:696-704.

37. Nagahara T, Yoshimatsu S, Shiba H, Kawaguchi H, Takeda K. Introduction of a mixture of $\beta$-tricalcium phosphate into a complex of bone marrow mesenchymal stem cells and type I collagen can augment the volume of alveolar bone without impairing cementum regeneration. J Periodontol. 2015;86:456-64.

38. Yuan J, Cui L, Zhang WJ, Liu W, Cao Y. Repair of canine mandibular bone defects with bone marrow stromal cells and porous beta- tricalcium phosphate. Biomaterials. 2007;28:1005-13.

39. Neamat A, Gawish A, Gamal-Eldeen A. $\beta$-tricalcium phosphate promotes cell proliferation, osteogenesis and bone regeneration in intrabony defects in dogs. Arch Oral Biol. 2009;54:1083-90.

40. Hasegawa N, Kawaguchi H, Hirachi A. Behaviorof transplanted bone marrow-derived mesenchymal stem cells in periodontal defects. J Periodontol. 2006;77:1003-07. 